(C) 1998 OPA (Overseas Publishers Association) N.V. Published by license under

the Harwood Academic Publishers imprint, part of The Gordon and Breach Publishing Group.

\title{
HIGH RESOLUTION SPECTROSCOPY WITH MOLECULAR BEAMS AND TUNABLE LASERS
}

\author{
R. VETTER*, P. LUC and C. AMIOT \\ Laboratoire Aimé Cotton ${ }^{\dagger}$, C. N. R. S. II, Bât.505, \\ 91405 Orsay Cedex, France
}

(Received 14 December 1997)

High resolution Doppler-free laser techniques are used in beam experiments to improve the spectroscopic description of complex diatomic molecules. The case of TiO is considered here for its implication in reaction dynamics studies and its interest in Astrophysics. Two absorption bands in the visible have been analyzed: $B^{3} \Pi-X^{3} \Delta(1-0)$ and $c^{1} \Phi-a^{1} \Delta(0-0)$. Owing to accurate wavenumber measurements, it has been possible to extend the analysis to high rotational quantum numbers and to carry out detailed spectroscopic calculations. They show that a careful revisiting of the TiO electronic structure is necessary.

Keywords: Tunable lasers; molecular beams; high resolution spectroscopy; TiO

\section{INTRODUCTION}

In the field of molecular physics, high resolution spectroscopy experiments offer a sharp contrast with time-resolved ones, as described by Zewail [1]. Actually, for decades, the search for high resolution was the wish and the will of experimentalists, with the wellknown consequences on modern physics. Nowadays, the state-of-theart in usual molecular spectroscopy is provided by Fourier Transform techniques whose etendue and multiplex advantages are decisive in a

\footnotetext{
*Corresponding author.

${ }^{\dagger}$ Laboratoire associé à l'Université Paris XI.
} 
number of areas $[2,3]$. Let us mention for instance the recordings of planet and star spectra which led to a breakthrough in Astrophysics [4] or the recording of the absorption spectrum of the iodine molecule whose Atlas is utilized by so many laser scientists to calibrate laser frequencies [5].

Usual molecular spectroscopy with Fourier Transform techniques leads to the simultaneous recording of a great many spectral elements, hence the description of the upper states in absorption spectroscopy and the description of lower states in excitation spectroscopy, i.e., when a laser beam is used to induce the fluorescence of the sample [6, 7]. However, these spectra are generally Doppler-limited, preventing their complete resolution when they are highly congested.

In contrast, by use of tunable narrow-band lasers, it is possible to get rid of the Doppler broadening. This was demonstrated years ago in atomic physics with two-photon absorption or saturated-absorption techniques, just to mention the most popular ones [8]. In molecular physics, these techniques and others, such as the optical-optical double resonance, led to a great simplification of the spectra, a much better knowledge of excited states and the discovery of hidden phenomena [9]. Let us mention for instance the recent study of very long-range sodium molecules [10] or the observation of hyperfine structures in photoassociative spectra of lithium [11].

In this article, Doppler-free experiments involving tunable laser beams and molecular beams are described to show how the description of complex diatomic molecules can be improved. The case of the TiO molecule is considered here, for its implication in reaction dynamics studies and its interest in Astrophysics.

\section{EXPERIMENTAL}

Accurate spectroscopic data are necessary when one wants to characterize chemical reaction products in the gas phase by use of optical detection techniques, such as laser-induced fluorescence (L.I.F.) resonance-enhanced multiphoton ionization (R.E.M.P.I.) or coherent anti-Stokes Raman scattering (C.A.R.S.) whose principle 
and application are easily found in the literature $[9,12,13]$. In the case of the $\mathrm{Ti}+\mathrm{O}_{2} \rightarrow \mathrm{TiO}+\mathrm{O}$ reaction, the lack of accurate experimental data about the $\mathrm{TiO}$ molecule is such that a high resolution analysis of its spectrum is obligatory.

In this perspective, a beam experiment has been devoted to record the Doppler-free absorption spectrum of TiO, by collecting the nondispersed laser-induced fluorescence of the molecule $v s$ laser frequency. It mainly involves an effusive beam of $\mathrm{TiO}$ crossed at right angle by a $\mathrm{cw}$, single-mode, tunable dye laser beam. The beam of TiO is produced in a tungsten crucible radiatively heated at $2,200 \mathrm{~K}$ [14]. It yields a density of $10^{8}-10^{9} \mathrm{~mol} / \mathrm{cm}^{3}$ at the interaction volume. The laser beam operating in the green-orange part of the spectrum is chopped at $750 \mathrm{~Hz}$ and delivers a typical power of $100 \mathrm{~mW}$ at the interaction volume. The induced fluorescence, collected by a large angle parabolic mirror whose focus coincides with the interaction volume, is sent to a photomultiplier through broad-band optical filters and is recorded $v s$ laser frequency by use of a lock-in amplifier at $750 \mathrm{~Hz}$. This method allows to eliminate parasitic signals such as the black body radiation emitted by the oven at high temperature. The signal is further stored in a PC disk, translated into ASCII and transmitted to a SPARC server for numerical treatment.

By slowly scanning the laser frequency over absorption lines of TiO, one records narrow profiles whose width, 3 to $510^{-3} \mathrm{~cm}^{-1}$ F.W.H.M., is determined by the lifetime of the upper level, by a residual Doppler broadening due to the small angular dispersion of the TiO beam and by weak saturation effects (see Figs. 1 and 2). To control the regularity of the frequency scale and to determine absolute $\mathrm{TiO}$ wavenumbers, the absorption spectrum of the iodine molecule and interference fringes provided by a Fabry-Perot etalon are simultaneously recorded and treated by numerical techniques [15].

This kind of experiment has been developed for long but seldom applied to systematic analyses of vibrational bands in complex spectra. The main advantages of the present experiment lie in the linearity of detection, the high resolution and the accuracy of wavenumber measurements which can reach $10^{-3} \mathrm{~cm}^{-1}$. They have allowed for extended analyses of the $\mathrm{TiO}$ spectrum which is particularly congested, as discussed in the following chapter. 


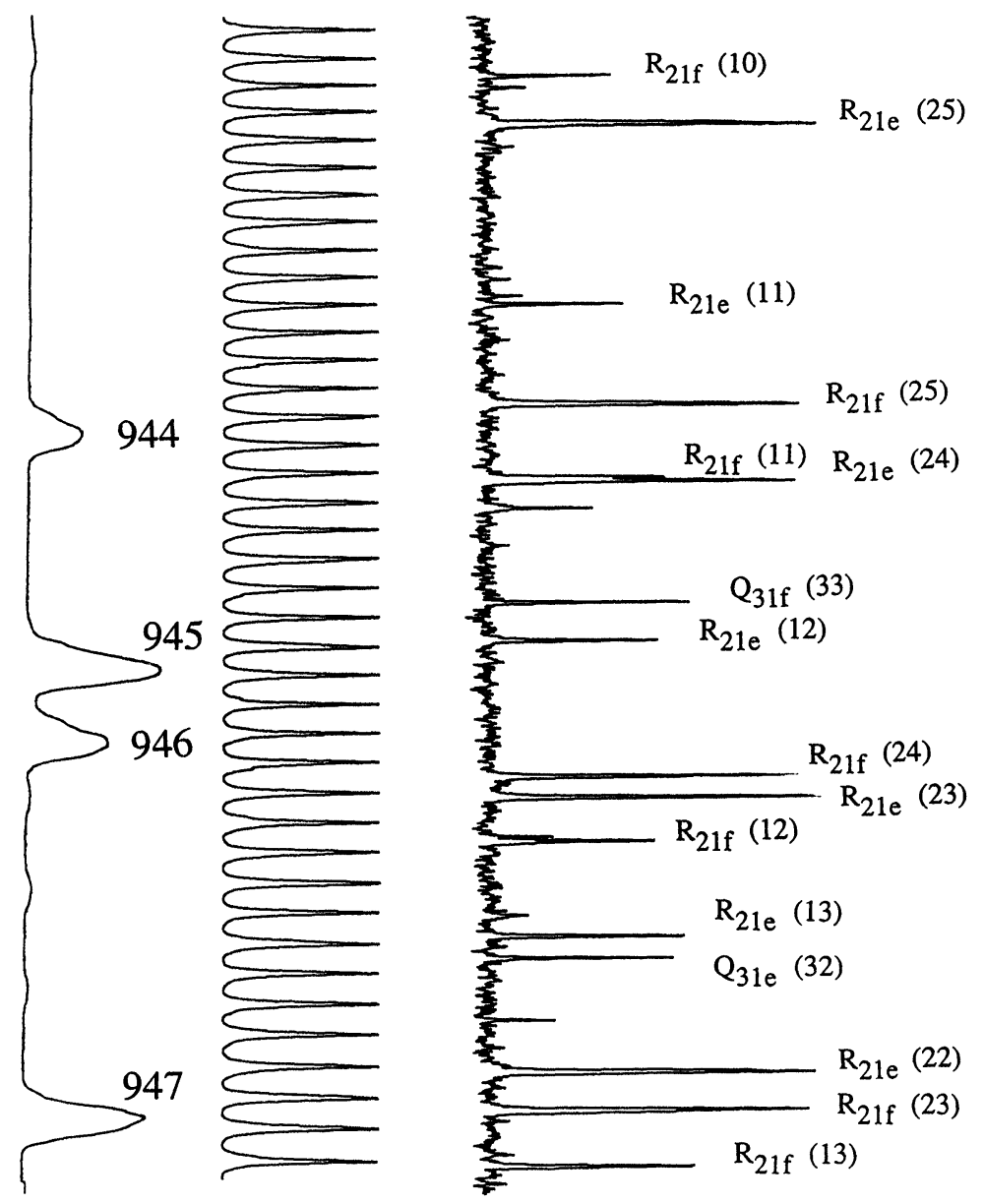

FIGURE 1 The $B^{3} \Pi-X^{3} \Delta(1-0)$ absorption band of TiO around $17,121 \mathrm{~cm}^{-1}$ (upper trace). Fabry-Perot reference fringes $2510^{-3} \mathrm{~cm}^{-1}$ apart (middle trace) and iodine absorption lines (lower trace) are used to calibrate the frequency scale. Iodine lines are identified from their reference number in the Atlas [5]. All intense lines are identified as belonging to the $B^{3} \Pi-X^{3} \Delta(1-0)$ band of ${ }^{48} \mathrm{TiO}$. The others belong to different systems or to different isotopomers.

\section{THE TiO SPECTRUM}

The TiO molecule has been the subject of several theoretical analyses since it constitutes a model for discussing the role of $3 d$ electrons in chemical bonding [16-18] and its absorption spectrum in the visible is 

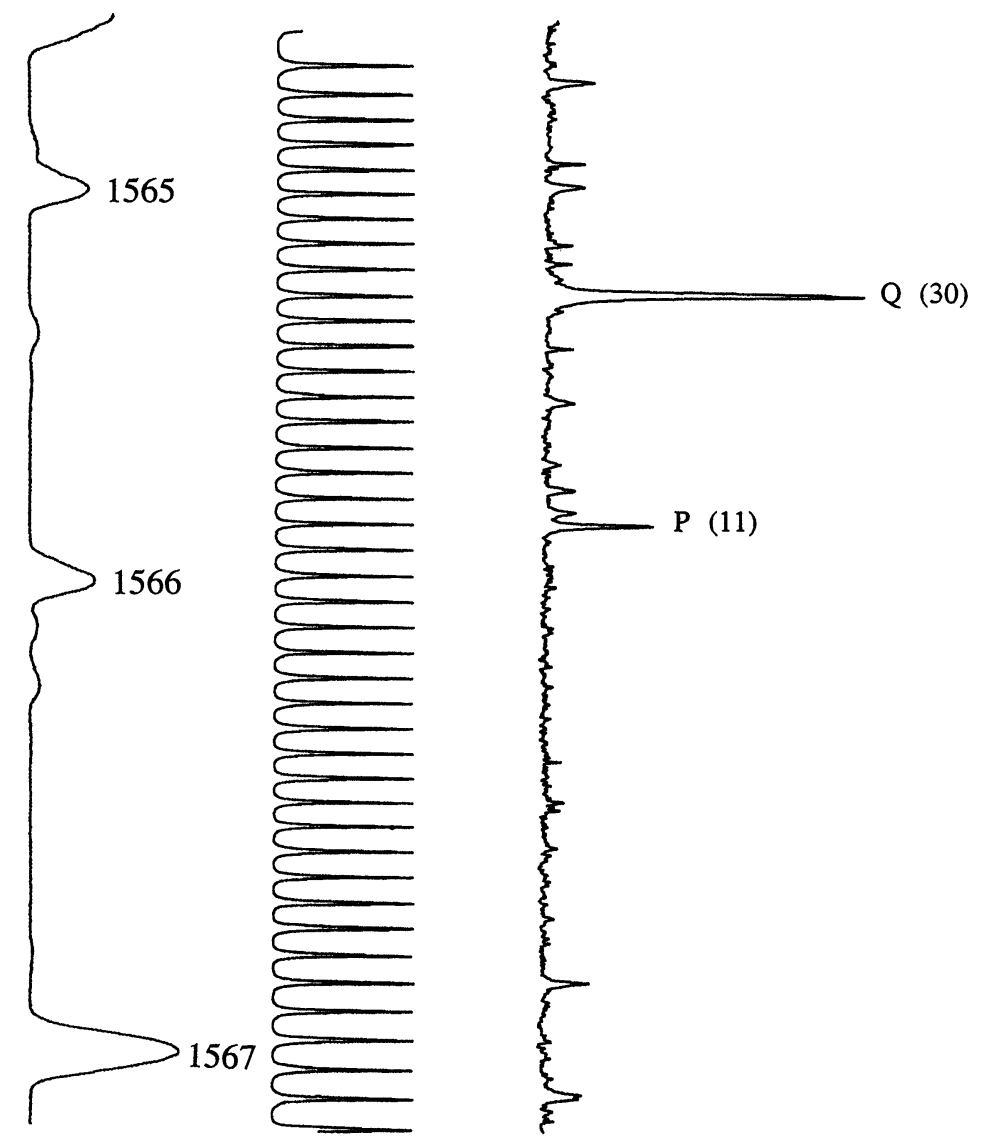

FIGURE 2 The $c^{1} \Phi-a^{1} \Delta(0-0)$ absorption band of TiO around $17,827 \mathrm{~cm}^{-1}$. Same captions as in Figure 1, except the laser intensity which is higher and leads to a weak broadening of the profiles.

still receiving much attention [19-27]. This is partly due to the fact that as $\mathrm{TiO}$ is abundant in oxygen-rich stars-the cool giants particularly-its spectrum is used to calibrate the temperature and density of these stars as soon as the rotational structure is resolved [28-31]. A number of low electronic states - singlets and triplets-have been discovered and rovibrational analyses have been carried out in spite of the highly congested character of the absorption spectrum and 
the limited resolution due to Doppler broadening. Indeed, in addition to usual rotational and spin-orbit structures, the levels in excited triplet states are split into e and $\mathrm{f}$ sub-levels due to $\Lambda$-doubling [32]. Furthermore, titanium has five natural isotopes which lead to five different absorption spectra, among which the one related to ${ }^{48} \mathrm{TiO}$ is prominent $(74 \%)$. This complexity is well illustrated by the Fourier Transform study which was performed several years ago by use of a hollow cathode as light source [23]. It significantly improved the description of the $X^{3} \Delta$ ground state by observing six $B^{3} \Pi-X^{3} \Delta$ and four $C^{3} \Delta-X^{3} \Delta$ bands but, due to Doppler broadening, the line assignation was limited to low values of the rotational quantum number, roughly $J^{\prime \prime}=50$. In this context, the aim of the present Doppler-free experiment is to extend the analysis to high rotational quantum numbers and to resolve isotope shifts. Two absorption bands have been treated: $B^{3} \Pi-X^{3} \Delta(1-0)$ and $c^{1} \Phi-a^{1} \Delta(0-0)$.

\section{The $B^{3} \Pi-X^{3} \Delta(1-0)$ Band [26] (Fig. 1)}

For this band, 1,400 lines were measured in the range 16,800-17, $200 \mathrm{~cm}^{-1}$ to a typical accuracy of $210^{-3} \mathrm{~cm}^{-1}$. Due to spin-orbit coupling in the two states and $\Lambda$-doubling in the $B$ one, 18 principal branches and 24 satellite ones are allowed. For the most abundant isotope of titanium the rotational analysis was conducted up to $J=94$. Spectroscopic data were reduced to a set of 24 effective molecular constants using a Hund's case (a) Hamiltonian. An iterative nonlinear least-square procedure was used and led to a standard deviation of $210^{-3} \mathrm{~cm}^{-1}$ between measured and fitted wavenumbers, to compare with $510^{-3} \mathrm{~cm}^{-1}$ obtained in the previous Fourier Transform analysis [23].

The rotational, spin-orbit and $\Lambda$-doubling constants deduced that way show interesting features in terms of electronic structures. Indeed, $a b$ initio calculations of the low-lying states of $\mathrm{TiO}[16,17]$ indicate that the electronic configuration which gives rise to the $X$ ground state is ... $(8 \sigma)^{2}(3 \pi)^{4}(9 \sigma)^{1}(1 \delta)^{1}$. By comparison, the configuration of the $B$ state is predicted to be $\ldots(8 \sigma)^{2}(3 \pi)^{4}(1 \delta)^{1}(4 \pi)^{1}$ by promotion of the $9 \sigma$ electron into a $4 \pi$ one [16]. These structures are confirmed to first order. To second order, spin-orbit effects result from singlet-triplet 
interactions between states coming from the same configuration, the $X^{3} \Delta$ and $a^{1} \Delta$ states for the $\sigma \delta$ configuration and the $B^{3} \Pi$ and ${ }^{1} \Pi$ states for the $\delta \pi$ configuration. The deviation to this scheme is weak for the $X$ state but not for the $B$ one. For this state, a supplementary interaction should occur with a new ${ }^{1} \Pi$ state which should lie below the $B$ state, by some $14,000 \mathrm{~cm}^{-1}$. This result is apparently meaningless since no ${ }^{1} \Pi$ state is present at this energy. In addition, the $\Lambda$ doubling in the $B^{3} \Pi$ state is also pertubed. For TiO, it should be due to ${ }^{3} \Sigma^{-}$and ${ }^{1} \Sigma^{+}$states arising from a $(1 \delta)^{2}$ configuration. The positions of these two states were predicted but their introduction into the interaction matrix elements leads once again to a meaningless result since then the spin-orbit operator takes half the correct value. Accordingly, it seems reasonable to foresee the revisiting of the $B^{3} \Pi$ state electronic structure.

For this band, the rather high signal/noise allows for the study of less intense components relative to less abundant titanium isotopes. The difficulty arises here from the weak, quasi-equal abundances of ${ }^{46} \mathrm{TiO},{ }^{47} \mathrm{TiO},{ }^{49} \mathrm{TiO}$ and ${ }^{50} \mathrm{TiO}$ whose entire spectra are shifted with respect to ${ }^{48} \mathrm{TiO}$ by several wavenumbers. These shifts can be evaluated, essentially by $a b$ initio mass corrections. To experimentally confirm them, it is best first to observe a branch head with its characteristic shape in a "desertic" spectral range and second to observe the $\Lambda$-doubling in the same range. For a complete assignment a long series of rotational quantum numbers is needed, implying the continuous recording of the spectrum over a wide spectral range, $25 \mathrm{~cm}^{-1}$ typically. Here, the rather good linearity of the L.I.F. technique of detection as well as the Doppler-free nature of the absorption profiles are favorable arguments. The complete analysis is on the tracks, the shifts being determined for ${ }^{46} \mathrm{TiO}$ and ${ }^{47} \mathrm{TiO}$ [33].

\section{The $c^{1} \Phi-a^{1} \Delta(0-0)$ Band [27] (Fig. 2)}

This band is simpler with $P, Q$ and $R$ branches only. It has by far the highest Franck-Condon factor of the $c^{1} \Phi-a^{1} \Delta$ system, implying that absorption of the laser beam and detection of the signal have to be performed at the same wavelength. However, the fluorescence signal is weak because of the small population in the $a^{1} \Delta\left(v^{\prime \prime}=0\right)$ metastable 
level which lies at $3,444 \mathrm{~cm}^{-1}$ above ground state. Therefore, a more intense laser beam was used, with a slight saturation of the absorption and a weak broadening of the profiles. Owing to the recent numerical treatment of the laser frequency scale [15] the accuracy of wavenumber measurements could be reduced to $10^{-3} \mathrm{~cm}^{-1}$ for 130 lines of ${ }^{48} \mathrm{TiO}$ measured in the range $17,700-17,860 \mathrm{~cm}^{-1}$ and the rotational analysis could be conducted up to $J=96$. The spectroscopic data were reduced to a set of 11 effective molecular constants with a standard deviation of $0.710^{-3} \mathrm{~cm}^{-1}$ between measured and fitted wavenumbers, to compare with $2010^{-3} \mathrm{~cm}^{-1}$ obtained in an older analysis performed with a Doppler-limited light source [21]. During the analysis, it was noted that the $\mathrm{H}$ constant $\left(3^{\text {rd }}\right.$ order centrifugal distortion) is abnormally high and negative and it was necessary to include significative seventh order constants into the energy development to reproduce the wavenumbers to their experimental accuracy. This explains why the extrapolation procedure was particularly difficult in the assignation of high $J$ values.

The spectroscopic treatment considered first the spin-orbit perturbation in the $c^{1} \Phi\left(v^{\prime}=0\right)$ level due to the neighbouring $C^{3} \Delta_{3}(v=2)$ level (about $100 \mathrm{~cm}^{-1}$ ). This was realized with the introduction of an enlarged Hamiltonian matrix in which a spin-orbit element $\mathrm{H}_{\mathrm{so}_{2}}$ couples the $\Omega=3$ sublevels of $C^{3} \Delta_{3}(v=2)$ and $c^{1} \Phi\left(v^{\prime}=0\right)$. This treatment was unsuccessful since it led to a r.m.s. of $9 \mathrm{~cm}^{-1}$, unless the 11 previous constants were introduced into the calculation. In a second step, the spin-orbit perturbation due to the much more distant $C^{3} \Delta_{3}(v=3)$ level (about $800 \mathrm{~cm}^{-1}$ ) was introduced. The same procedure was used, with the introduction of a new spin-orbit matrix element $\mathrm{H}_{\mathrm{so}_{3}}$ coupling the $\Omega=3$ sublevels of $C^{3} \Delta_{3}(v=3)$, $C^{3} \Delta_{3}(v=2)$ and $c^{1} \Phi\left(v^{\prime}=0\right)$. Then, the total perturbation is characterized by a unique electronic factor $H_{\text {so elec }}$ equal to the product of $\mathrm{H}_{\mathrm{sO}_{3}}$ by an overlap element calculated from RKR potentials. With this $\mathrm{H}_{\text {so elec }}$ factor, the r.m.s. falls to $0.810^{-3} \mathrm{~cm}^{-1}$ which is comparable with the experimental accuracy. However, once again, the $\mathrm{H}_{\text {so elec }}$ factor which is determined this way is not in agreement with the one derived from ab initio calculations.

The ability of this deperturbed treatment to yield meaningful molecular constants is of interest in Astrophysics where high values of rotational quantum numbers are often required [31]. 


\section{DISCUSSION}

The accuracy in wavenumber measurements mentioned above $-10^{-3} \mathrm{~cm}^{-1}-$ is neither limited by the width and shape of TiO absorption profiles, nor by the signal/noise but by the asymmetry of iodine absorption lines used to calibrate the laser frequency scale [15]. To get rid of this asymmetry due to hyperfine structure, one could undertake complex, Doppler-free experiments to resolve the structure, to calibrate the individual components and to rebuilt the whole Doppler-broadened profile. The alternative which consists in using the bromine Atlas is no longer convenient because it requires highly enriched isotopes and furthermore the fluorescence is much less intense. Actually, the $10^{-3} \mathrm{~cm}^{-1}$ accuracy presently obtained is fairly enough but necessary both to resolve the spectrum and to perform accurate spectroscopic calculations. If it seems to constitute a benchmark for molecular spectroscopy, at least for band analysis, it is still needed when one is faced to high resolution experiments: indeed, for a light molecule like $\mathrm{Li}_{2}$, the discrepancy between the wavenumbers measured in the same beam experiment and the ones calculated from the best known spectroscopic constants can easily range from 10 to 30 $10^{-3} \mathrm{~cm}^{-1}$ depending on the band under study [34].

Of course, there are many examples of better accuracy but they generally concern the study of single lines or groups of lines in limited spectral ranges [9] not the analysis of vibrational bands. Table I displays some characteristic results on band analysis of diatomic molecules, including TiO, obtained with the same detection technique and frequency calibration. Note the excellent result obtained by Demtröder and coll. as early as 1979, with polarization spectroscopy of the $B^{1} \Pi_{u}-X^{1} \Sigma_{g}^{+}$band of $\mathrm{Cs}_{2}$ [35] and the most accurate one obtained by Merer and coll. on a $B^{3} \Phi-X^{3} \Delta$ band of $\mathrm{NbN}$, with hyperfine structure measurements over 1,000 transitions [36]. Note also the rather good accuracy obtained by Kaledin and coll. over weak singlet - triplet interconnections lines of TiO [25].

Regarding the TiO spectrum itself, the present results are by far the most accurate ones. By comparison with Linton's work performed long ago over the same $c^{1} \Phi-a^{1} \Delta(0-0)$ band [21] not only the band heads are now completely resolved and the r.m.s. is lower by a factor 15 , but the analysis has been performed up to $J=96$ instead of 70 . 
TABLE I Several high accuracy rotational analyses in diatomic spectra (nonexhaustive list)

\begin{tabular}{lccc}
\hline Molecule & Transition & $\begin{array}{c}\text { r.m.s. } \\
\left(10^{-3} \mathrm{~cm}^{-1}\right)\end{array}$ & Author, date, reference \\
\hline $\mathrm{Cs}_{2}$ & $B^{1} \Pi_{u}-X^{1} \Sigma_{g}^{+}$ & 1 & Demtröder et al., $1979[35]$ \\
$\mathrm{CrO}$ & $A^{2} \Sigma-X^{2} \Pi^{+}$ & 6.7 & Merer et al., $1984[36]$ \\
$\mathrm{IF}$ & ${ }^{3} \Pi\left(\mathrm{O}^{+}\right)-X^{1} \Sigma^{+}$ & 4.3 & Vigué et al., $1987[38]$ \\
$\mathrm{NbN}$ & $B^{3} \Phi-X^{3} \Delta$ & 0.6 & Merer et al., $1989[37]$ \\
$\mathrm{CaO}$ & $e^{3} \Sigma^{-}-a^{3} \Pi$ & 3 & Field et al., 1991 [39] \\
$\mathrm{BaI}$ & $C^{2} \Pi-X^{2} \Sigma^{+}$ & 2.3 & Zare et al., 1992 [40] \\
$\mathrm{CrO}$ & $A^{\prime 5} \Delta-X^{5} \Pi$ & 1.3 & Merer et al., $1993[41]$ \\
$\mathrm{TiO}$ & $E^{3} \Pi-X^{3} \Delta$ & 20 & Simard et al., 1991 [24] \\
TiO & $C^{3} \Delta-a^{1} \Delta$ & 10 & Kaledin et al., 1995 [25] \\
TiO & $B^{3} \Pi-X^{3} \Delta$ & 2 & Amiot et al., $1995[26]$ \\
TiO & $c^{1} \Phi-a^{1} \Delta$ & 0.7 & Amiot et al. . $1996[27]$ \\
\hline
\end{tabular}

\section{Acknowledgements}

The authors are pleased to thank E. M. Azaroual and M. Cheikh for their participation.

\section{References}

[1] Zewail, A. H. (1995). "Femtosecond Chemistry", Eds. Manz, J. and Wöst, L. Pub. $\mathrm{VCH}$ press.

[2] Jacquinot, P. (1958). J de Physique et le Radium (Paris) 19, 223.

[3] Amiot, C. and Vergès, J. (1987). J. de Physique (Paris), 12, 595.

[4] Connes, P., Connes, J., Benedict, W. S. and Kaplan, L. D. (1967). Astrophys. J., 147, 1231; Connes, P. and Connes, J. (1974). Astrophys. J., L-29.

[5] Gerstenkorn, S. and Luc, P. (1977). Atlas d'absorption de la molécule d'iode, Laboratoire Aimé Cotton, Orsay.

[6] Bacis, R., Churrassy, S., Field, R. W., Koffend, J. B. and Vergès, J. (1980). J. Chem. Phys., 72, 34.

[7] Vergès, J., Amiot, C., Bacis, R. and Ross, A. J. (1995). Spectroc. Acta, 51A, 1191.

[8] High Resolution Laser Spectroscopy, Ed. Shimoda, K. Springer-Verlag (Berlin) (1976).

[9] Demtröder, W. (1995). in Laser Spectroscopy, Springer-Verlag (Berlin).

[10] Elbs, M., Keck, O., Knöckel, H. and Tiemann, E., Z. Physik D, 42, 49.

[11] Abraham, E. R. I., McAlexander, W. I., Stoof, H. T. C. and Hulet, R. G. (1995). Phys. Rev. A, 53, 3092.

[12] Atomic and Molecular Beam Methods, Vol. I and II, Ed. Scoles, G. Oxford University Press (Oxford) (1992).

[13] Gonzalez-Urena, A. and Vetter, R. (1996). Int. Rev. Phys. Chem., 15, 375.

[14] Azaroual, E. M., Luc, P. and Vetter, R. (1992). J. de Physique III (Paris), 2, 899.

[15] Luc, P., d'Aignaux, Y., Amiot, C. and Vetter, R. (1997). Metrologia, 34, 319.

[16] Carlson, K. D. and Nesbet, R. K. (1964). J. Chem. Phys., 41, 1051; Carlson, K. D. and Moser, C. (1967). J. Chem. Phys., 46, 35.

[17] Bauschlicher, C. W.Jr., Bagus, P. S. and Nelin, C, J. (1983). Chem. Phys. Lett., 101, 229. 
[18] Sennesal, J. M. and Schamps, J. (1987). Chem. Phys., 114, 37.

[19] Huber, K. P. and Herzberg, G. (1979). Constants of Diatomic Molecules, Van Nostrand-Reinhold, Princeton.

[20] Phillips, J. G. (1973). Astrophys. J. Suppl. Series, 26, 313.

[21] Linton, C. (1974). J. Mol. Spectrosc., 50, 235; Linton, C. and Broida, H. P. (1985). J. Mol. Spectrosc., 109, 345.

[22] Hocking, W. H., Gerry, M. C. L. and Merer, A. J. (1979). Can. J. Phys., 57, 54; Merer, J. (1989). Annu. Rev. Phys. Chem., 40, 407; Barnes, J., Merer, A. J. and Metha, G. F. (1996). J. Mol. Spectrosc., 180, 437.

[23] Gustavsson, T., Amiot, C. and Vergès, J. (1991). J. Mol. Spectrosc., 145, 56.

[24] Simard, B. and Hackett, P. A. (1991). J. Mol. Spectrosc., 148, 128.

[25] Kaledin, L. A., Cord, J. E. and Heaven, M. C. (1995). J. Mol. Spectrosc., 173, 499.

[26] Amiot, C., Azaroual, E. M., Luc, P. and Vetter, R. (1995). J. Chem. Phys., 102, 4375.

[27] Amiot, C., Cheikh, M., Luc, P. and Vetter, R. (1996). J. Mol. Spectrosc., 179, 159.

[28] Merrill, P. W., Deutsch, A. J. and Keenan, P. C. (1962). Astrophys. J., 136, 21.

[29] Phillips, J. G. (1952). Astrophys. J., 115, 183.

[30] Phillips, J. G. and Davis, S. P. (1987). Publ. Astron. Soc. Pac., 99, 839.

[31] Lambert, D. L. (1994). in Molecules in the Stellar Environment, Ed. Jorgensen, U. G., Springer-Verlag, Berlin.

[32] Herzberg, G. (1950). Molecular Spectra and Molecular Strucutre, Vol. I, Van Nostrand, New York.

[33] Amiot, C., Cheikh, M., Luc, P. and Vetter, R. (1998). to be published.

[34] Cacciani, P. (1997). Laboratoire Aimé Cotton, Orsay, private comm.

[35] Raab, M., Höning, G., Castell, R. and Demtröder, W. (1979). Chem. Phys. Lett., 66, 307.

[36] Cheung, A. S. C., Zernicki, W. and Merer, A. J. (1984). J. Mol. Spectrosc., 104, 315.

[37] Azuma, Y., Barry, J. A., Lyne, M. P. J., Merer, A. J., Schröder, J. O. and Feminias, J. L. (1989). J. Chem. Phys., 91, 1.

[38] Gouédard, G., Billy, N., Girard, B. and Vigué, J. (1987). Mol. Phys., 62, 1371.

[39] Baldwin, D. P., Norman, J. B., Soltz, R. A., Sur, A. and Field, R. W. (1991). J. Mol. Spectrosc., 139, 39.

[40] Leach, C. A., Tsekouras, A. A. and Zare, R. N. (1992). J. Mol. Spectrosc., 153, 59.

[41] Barnes, M., Hajigeorgiou, P. G. and Merer, A. J. (1993). J. Mol. Spectrosc., 160, 289. 